\title{
A study on compactness in metric spaces and topological spaces
}

\author{
Rabeya Akter ${ }^{1}$, Nour Mohammed Chowdhury², Mohammad Safi Ullah ${ }^{3}$ \\ ${ }^{1}$ Department of Mathematics, Jagannath University, Dhaka, Bangladesh \\ ${ }^{2}$ Department of Mathematics, World University of Bangladesh, Dhaka, Bangladesh \\ ${ }^{3}$ Department of Mathematics, Comilla University, Comilla, Bangladesh
}

Email address:

rabeya.ju@gmail.com (R. Akter), ranapu06@gmail.com, (N. M. Chowdhury), Safi.ru1985@gmail.com (M. S. Ullah)

\section{To cite this article:}

Rabeya Akter, Nour Mohammed Chowdhury, Mohammad Safi Ullah. A Study on Compactness in Metric Spaces and Topological Spaces. Pure and Applied Mathematics Journal. Vol. 3, No. 5, 2014, pp. 105-112. doi: 10.11648/j.pamj.20140305.13

\begin{abstract}
Topology may be considered as an abstract study of the limit point concept. As such, it stems in part from recognition of the fact that many important mathematical topics depend entirely upon the properties of limit points. This study shows that compactness implies limit point compactness but not conversely and every compact space is locally compact but not conversely. This study also shows that compactness, limit point compactness and sequentially compactness are equivalent in metrizable spaces and the product of finitely many compact spaces is a locally compact space. This study introduce it here as an interesting application of the Tychonoff theorem.
\end{abstract}

Keywords: Metric Spaces, Topological Space, Compact Space, Locally Compact Space, Sequentially Compactness, Neighborhood

\section{Introduction}

The theory of topological spaces or, as it is called, point set or general topology, has become one of the elementary building blocks underlying diver's branches of mathematics. Its concepts and methods have enriched numerous other fields of mathematics and given considerable impulse to their further development. For these reasons, topology counts among those few basic structures, which alone give access to modern mathematical research.

In this work, we have tried to accumulate several types of concepts of separation axioms on topological space, obtained from different texts. Numerous proofs, examples and solved problems are included in this work. Up to now, no work ever complete. We only try to discuss the clear concepts of compactness on topological spaces, its properties and applications, so that one can see these materials concerning compactness on topological spaces at one place.

\subsection{Objective}

The advantage in compact spaces is that one may study the whole space by studying a finite number of open subsets. We shall see this when we prove that a continuous function $f$ :
$\mathrm{X} \rightarrow \mathrm{Y}$ from a compact metric space $\mathrm{X}$ to a metric space $\mathrm{Y}$ is "uniformly continuous." In conclusion we shall examine some compact surfaces that may be formed by "identifying" edges of a rectangle.

\subsection{Procedure}

In the proposed study, first, we shall give some necessary definitions and states some necessary theorems in order to present the paper in a self contained manner. Afterward, we shall prove the most important and popular theorem namely Heine-Borel Theorem. Then we shall study locally compact spaces of a metric space and in general of a topological space. Next, we shall discuss $\sigma$-compact and finally compact spaces and some of their important properties. Finally, we shall study completely compact spaces, anti-compact spaces and completely dense subsets in a space and some of their important properties.

\section{Definition:}

Let $\left\{G_{i}\right\}$ be a class of subsets of $X$ such that $A \subset \cup_{i} G_{i}$ for some $A \subset X$. The class $\left\{G_{i}\right\}$ is then called a cover of $A$, and an open cover if each $G_{i}$ is open. Furthermore, if a finite subclass of $\left\{G_{i}\right\}$ is also a cover of A, i.e. if $\exists$ $\mathrm{G}_{\mathrm{i}_{1}}, \mathrm{G}_{\mathrm{i}_{2}}, \ldots \ldots \ldots \ldots \ldots \ldots \ldots, \mathrm{G}_{\mathrm{i}_{\mathrm{m}}} \in\left\{\mathrm{G}_{\mathrm{i}}\right\}$ such that $\mathrm{A} \subset$ 
$\mathrm{G}_{\mathrm{i}_{1}} \cup \mathrm{G}_{\mathrm{i}_{2}} \cup \ldots \ldots \ldots \ldots \ldots \ldots \ldots . . . . . . . . . . . \mathrm{G}_{\mathrm{i}_{\mathrm{m}}}$, then $\left\{\mathrm{G}_{\mathrm{i}}\right\}$ is said to be reducible to a finite cover, or contains a finite subcover.

\subsection{Compact Sets}

A subset $\mathrm{A}$ of a topological space $(\mathrm{X}, \mathrm{T})$ is compact if every open cover of $\mathrm{A}$ is reducible to a finite subcover.

In other words, if $A$ is compact and $A \subset \bigcup_{i} G_{i}$, where $G_{i}$ are open sets, then one can select a finite number of the open sets, say $G_{i_{1}}, G_{i_{2}}, \ldots \ldots \ldots \ldots \ldots \ldots \ldots \ldots . . . G_{i_{m}}$, so that $A \subset$

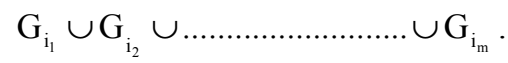

\subsection{Compact Spaces}

A topological space $\mathrm{X}$ is said to be compact if for each open covering $\left(\mathrm{U}_{\alpha}\right)_{\alpha \in \mathrm{I}}$ of $\mathrm{X}$ there is a finite subcovering $\left(\mathrm{V}_{\beta}\right)_{\beta \in \mathrm{J}}$

We may alternatively define compactness by the statement, " $\mathrm{X}$ is compact if for each open covering $\left(\mathrm{U}_{\alpha}\right)_{\alpha \in \mathrm{I}}$ of $\mathrm{X}$ there is a finite subset of indices $\left\{\alpha_{1}, \alpha_{2}, \ldots \ldots \ldots, \alpha_{n}\right\}$ such that the collection $U_{\alpha_{1}}, U_{\alpha_{2}}, \ldots \ldots \ldots . . ., U_{\alpha_{n}}$ covers X."

Definition:

A subset $\mathrm{C}$ of a topological space $\mathrm{X}$ is said to be compact, if $\mathrm{C}$ is a compact topological space in the relative topology.

Example:

The real line $\mathbb{R}$ is not compact, for the covering of $\mathbb{R}$ by open intervals

$\mathcal{A}=\{(\mathrm{n}, \mathrm{n}+2) \mid \mathrm{n} \in \mathbb{Z}\}$

contains no finite subcollection that covers $\mathbb{R}$.

Example :

Any space $\mathrm{X}$ containing only finitely many points is necessarily compact, because in this case every open covering of $\mathrm{X}$ is finite.

\section{Compactness}

Theorem 2.1 $1^{[6]}$ :

A subset $\mathrm{C}$ of a topological space $\mathrm{X}$ is compact if and only if for each open covering $\left(\mathrm{U}_{\alpha}\right)_{\alpha \in \mathrm{I}}$ of $\mathrm{C}, \mathrm{U}_{\alpha}$ open in $\mathrm{X}$, there is a finite subcovering $\mathrm{U}_{\alpha_{1}}, \ldots \ldots \ldots . ., \mathrm{U}_{\alpha_{\mathrm{n}}}$ of $\mathrm{C}$.

Proof: Let $\mathrm{C}$ be compact and let $\left(\mathrm{U}_{\alpha}\right)_{\alpha \in \mathrm{I}}$ be an open covering of $\mathrm{C}$. Thus $\mathrm{C} \subset \bigcup_{\alpha \in \mathrm{I}} \mathrm{U}_{\alpha}$, hence $\mathrm{C}=\bigcup_{\alpha \in \mathrm{I}}\left(\mathrm{U}_{\alpha} \cap C\right)$, so that the family $\left(\mathrm{U}_{\alpha} \cap \mathrm{C}\right)_{\alpha \in \mathrm{I}}$ is a covering of the topological space $C$ by relatively open sets $U_{\alpha} \cap C$. Since $C$ is compact, there is a finite collection of indices $\left\{\alpha_{1}, \alpha_{2}, \ldots ., \alpha_{n}\right\}$ such that $\mathrm{U}_{\alpha_{1}} \cap \mathrm{C}, \mathrm{U}_{\alpha_{2}} \cap \mathrm{C}, \ldots \ldots \ldots . ., \mathrm{U}_{\alpha_{\mathrm{n}}} \cap \mathrm{C}$ covers $\mathrm{C}$; that is $\mathrm{C}$ $=\bigcup_{i=1}^{\mathrm{n}}\left(\mathrm{U}_{\alpha_{i}} \cap \mathrm{C}\right) \cdot$ Consequently, $\mathrm{C} \subset \bigcup_{i=1}^{n} U_{\alpha_{i}}$.

Conversely, suppose that for each open covering $\left(\mathrm{U}_{\alpha}\right)_{\alpha \in \mathrm{I}}$ of $\mathrm{C}$, there is a finite subcovering $\mathrm{U}_{\alpha_{1}}, \mathrm{U}_{\alpha_{2}}, \ldots \ldots \ldots \ldots, \mathrm{U}_{\alpha_{\mathrm{n}}}$ of $\mathrm{C}$. We must show that given any covering of the topological space $\mathrm{C}$ by a family $\left(\mathrm{V}_{\beta}\right)_{\beta \in \mathrm{J}}$ of relatively open subsets of $\mathrm{C}$, there is a finite subcovering. For each $\beta \in \mathrm{J}$,

Since $V_{\beta}$ is relatively open in $C$, there is an open subset $U_{\beta}$ of $X$ such that $V_{\beta}=U_{\beta} \cap C$. But $C=\bigcup_{\beta \in J} V_{\beta}$, therefore $C \subset \bigcup_{\beta \in J} U_{\beta}$ and $\left(U_{\beta}\right)_{\beta \in J}$ is a covering of the subset $C$ by open sets. By our hypothesis, there is a finite subcovering $\mathrm{U}_{\beta}$,

$\mathrm{U}_{\beta}{ }_{2}, \ldots \ldots \ldots, \mathrm{U}_{\beta} \mathrm{m}$ of $\mathrm{C}$. Thus, $\mathrm{C} \subset \bigcup_{\mathrm{i}=1}^{\mathrm{m}} \mathrm{U}_{\beta_{\mathrm{i}}}$ and $\mathrm{C}=$ $\left(\left(\bigcup_{i=1}^{m} U_{\beta_{i}}\right) \cap C=\bigcup_{i=1}^{m}\left(U_{\beta_{i}} \cap C\right)=\bigcup_{i=1}^{m} V_{\beta_{i}}\right.$

Hence the covering $\left(\mathrm{V}_{\beta}\right)_{\beta \in J}$ of $\mathrm{C}$ by relatively open sets has a finite subcovering $\mathrm{V}_{\beta_{1}}, \mathrm{~V}_{\beta_{2}}, \ldots \ldots \ldots \ldots, \mathrm{V}_{\beta} \mathrm{m}$.

Compactness may be characterized in terms of neighbourhoods.

Theorem 2.2 $2^{[6]}$.

A topological space $\mathrm{X}$ is compact if and only if, whenever for each $x \in X$ a neighbourhood $N_{x}$ of $x$ is given, there is finite number of points

$\mathrm{x}_{1}, \mathrm{x}_{2}, \ldots \ldots, \mathrm{x}_{\mathrm{n}}$ of $\mathrm{X}$ such that $\mathrm{X}=\bigcup_{\mathrm{i}=1}^{\mathrm{n}} \mathrm{N}_{\mathrm{x}_{\mathrm{i}}} \cdot$

Theorem $2.3^{[4]}$ :

Any closed subspace of a compact space is compact.

Proof: Let $\mathrm{Y}$ be a closed of a compact space $\mathrm{X}$, and let $\left\{\mathrm{G}_{\mathrm{i}}\right\}$ be an open cover of $Y$. Each $G_{i}$, being open in the relative topology on $\mathrm{Y}$, is the intersection with $\mathrm{Y}$ of an open subset $\mathrm{H}_{\mathrm{i}}$ of $\mathrm{X}$. Since $\mathrm{Y}$ is closed, the class composed of $\mathrm{Y}^{\prime}$ and all the $H_{i}$ 's is an open cover of $X$, and since $X$ is compact, this open cover has a finite subcover. If $\mathrm{Y}^{\prime}$ occurs in this subcover, we discard it. What remains is a finite class of $\mathrm{H}_{\mathrm{i}}$ 's whose union contains $\mathrm{X}$. Our conclusion that $\mathrm{Y}$ is compact now follows from the fact that the corresponding $\mathrm{G}_{\mathrm{i}}$ 's form a finite subcover of the original open cover of $\mathrm{Y}$.

Theorem 2.4 $4^{[4]}$ :

Any continuous image of a compact space is compact.

Proof: Let $\mathrm{f}: \mathrm{X} \rightarrow \mathrm{Y}$ be a continuous mapping of a compact space $\mathrm{X}$ into an arbitrary topological space $\mathrm{Y}$. We must show that $\mathrm{f}(\mathrm{x})$ is a compact subspace of $\mathrm{Y}$. Let $\left\{\mathrm{G}_{\mathrm{i}}\right\}$ be an open cover of $f(x)$. As in the above proof, each $G_{i}$ is the intersection with $\mathrm{f}(\mathrm{X})$ of an open subset $\mathrm{H}_{\mathrm{i}}$ of $\mathrm{Y}$. It is clear that $\left\{\mathrm{f}^{-1}\left(\mathrm{H}_{\mathrm{i}}\right)\right\}$ is an open cover of $\mathrm{X}$, and by the compactness of $\mathrm{X}$ it has a finite subcover. The union of the finite class of $\mathrm{H}_{\mathrm{i}}$ 's of which these are the inverse images clearly contains $f(X)$, so the class of corresponding $G_{i}$ 's is a finite subcover of the original open cover of $f(X)$, and $f(X)$ is compact.

Theorem $2.5^{[3]}$ :

If $\mathrm{A}$ is a compact subset of a Hausdorff space $\mathrm{X}$ and $\mathrm{x}$ is a point of $\mathrm{X}-\mathrm{A}$, then there are disjoint neighbourhoods of $\mathrm{X}$ and of $\mathrm{A}$.

Consequently each compact subset of a Hausdorff space is closed.

Proof: Since X is Hausdorff there is a neighbourhood U of each point $\mathrm{y}$ of $\mathrm{A}$ such that $\mathrm{x}$ does not belong to the closure $\bar{U}$. Because $A$ is compact there is a finite family $U_{0}$, $\mathrm{U}_{1}, \ldots \ldots \ldots, \mathrm{U}_{\mathrm{n}}$ of open sets covering A such that $\mathrm{x} \notin \overline{\mathrm{U}}_{\mathrm{i}}$ for $\mathrm{i}=0,1, \ldots \ldots \ldots ., \mathrm{n}$. If $\left.\mathrm{V}=\mathrm{U}_{\{\mathrm{Ui}} \mathrm{i}=0,1, \ldots ., \mathrm{n}\right\}$, then $\mathrm{A} \subset \mathrm{V}$ and $\mathrm{x} \notin \overline{\mathrm{V}}$. Consequently $\mathrm{X}-\overline{\mathrm{V}}$ and $\mathrm{V}$ are disjoint neighbourhoods of $\mathrm{x}$ and $\mathrm{A}$. 


\section{Theorem $2.6^{[3]}$.}

Let $\mathrm{f}$ be a continuous function carrying the compact topological space $\mathrm{X}$ onto the topological space $\mathrm{Y}$. Then $\mathrm{Y}$ is compact, and if $\mathrm{Y}$ is Hausdorff and $\mathrm{f}$ is one to one then $\mathrm{f}$ is a homomorphism.

Proof: If $\alpha$ is an open cover of $\mathrm{Y}$, then the family of all sets of the form $\mathrm{f}^{-1}[\mathrm{~A}]$ for $\mathrm{A}$ in $\alpha$, is an open cover of $\mathrm{X}$ which has a finite subcover. The family of images of members of the subcver is a finite subfamily of $\alpha$ which covers $Y$ and consequently $\mathrm{Y}$ is compact. Suppose that $\mathrm{Y}$ is hausdorff and $\mathrm{f}$ is one to one. If $\mathrm{A}$ is a closed subset of $\mathrm{X}$, then $\mathrm{A}$ is compact and hence its image $\mathrm{f}[\mathrm{A}]$ is compact and therefore closed. Then $\left(\mathrm{f}^{-1}\right)^{-1}[\mathrm{~A}]$ is closed for each closed set $A$ and $\mathrm{f}^{-1}$ is continuous.

Theorem 2. $7^{[3]}$ :

If $\mathrm{A}$ and $\mathrm{B}$ are disjoint compact subsets of a Hausdorff space $\mathrm{X}$, then there are disjoint neighbourhoods of A and $\mathrm{B}$.

Consequeltly each compact Hausdorff space is normal.

Proof: For each $\mathrm{x}$ in A there is a neighbourhood of $\mathrm{x}$ and a neighbourhood of $\mathrm{B}$ which are disjoint. Consequently there is a neighbourhood $\mathrm{U}$ of $\mathrm{x}$ whose closure is disjoint from $\mathrm{B}$, and since $A$ is compact there is a finite family $U_{0}$, $\mathrm{U}_{1}, \ldots \ldots \ldots, \mathrm{U}_{\mathrm{n}}$ such that $\overline{\mathrm{U}}_{\mathrm{i}}$ is disjoint from $\mathrm{B}$ for $\mathrm{i}=1, \ldots \ldots ., \mathrm{n}$ and $\mathrm{A} \subset \mathrm{V}=\bigcup\left\{\mathrm{U}_{\mathrm{i}}: \mathrm{i}=0,1, \ldots \ldots, \mathrm{n}\right\}$. Then $\mathrm{v}$ is a neighbourhood of $\mathrm{A}$ and $\mathrm{X}-\overline{\mathrm{V}}$ is a neighborhood of $\mathrm{B}$ which is disjoint from $\mathrm{V}$.

Theorem $2.8^{[3]}$ :

If $\mathrm{X}$ is a regular topological space, A a compact subset, and $U$ a neighbourhood of $A$, then there is a closed neighbourhood $\mathrm{V}$ of $\mathrm{A}$ such that $\mathrm{V} \subset \mathrm{U}$.

Consequently each compact regular space is normal.

Proof: Because $\mathrm{X}$ is regular, for each $\mathrm{x}$ in $\mathrm{A}$ there is an open neighbourhood $\mathrm{W}$ of $\mathrm{x}$ such that $\overline{\mathrm{W}} \subset \mathrm{U}$, and by compactness there is a finite open cover $\mathrm{W}_{0}, \mathrm{~W}_{1}, \ldots \ldots \ldots$... $\mathrm{W}_{\mathrm{n}}$ of $\mathrm{A}$ such that $\overline{\mathrm{W}}_{\mathrm{i}} \subset \mathrm{U}$ for each $\mathrm{i}$. Then $\mathrm{V}=\bigcup\left\{\overline{\mathrm{W}}_{\mathrm{i}}: \mathrm{i}=0\right.$, $1, \ldots \ldots, \mathrm{n}\}$ is the required neighbourhood of $\mathrm{A}$.

Theorem 2.9 $9^{[3]}$ :

If $\mathrm{X}$ is a completely regular space, $\mathrm{A}$ is a compact subset and $U$ is a neighbourhood of $A$, then there is a continuous function $\mathrm{f}$ on $\mathrm{X}$ to the closed interval $[0,1]$ such that $\mathrm{f}$ is one on $\mathrm{A}$ and zero on $\mathrm{X}-\mathrm{U}$.

Proposition 2.1 $1^{[9]}$ :

For a topological space $\mathrm{X}$, the following are equivalent

(1) $\mathrm{X}$ is compact

(2) If $\mathcal{F}$ is any family of closed sets in $X$ with fip, then $\bigcap$ $\mathcal{F}=\phi$

(3) Every net in $X$ has a cluster point.

Theorem (The Heine-Borel Theorem) $2.10^{[4]}$ :

Every closed and bounded subspace of the real line is compact.

Proof: A closed and bounded subspace of the real line is a closed subspace of some closed interval $[a, b]$, it suffices to show that $[a, b]$ is compact. If $a=b$, this is clear, so we may assume that $\mathrm{a}<\mathrm{b}$. we know that the class of all intervals of the form $[\mathrm{a}, \mathrm{d})$ and $(\mathrm{c}, \mathrm{b}]$, where $\mathrm{c}$ and $\mathrm{d}$ are any real numbers such that $\mathrm{a}<\mathrm{c}<\mathrm{b}$ and $\mathrm{a}<\mathrm{d}<\mathrm{b}$, is an open subbase for $[\mathrm{a}, \mathrm{b}]$; therefore the class of all [a, c]'s and all [d, b]'s is a closed subbase. Let $\mathrm{S}=\left\{\left[\mathrm{a}, \mathrm{c}_{\mathrm{i}}\right],\left[\mathrm{d}_{\mathrm{j}}, \mathrm{b}\right]\right\}$ be a class of these subbasic closed sets with the finite intersection property. It suffices to show that the intersection of all sets in $\mathrm{S}$ is non-empty. We may assume that $\mathrm{S}$ is non-empty. If $\mathrm{S}$ contains only intervals of the type $\left[a, c_{i}\right]$, or only intervals of the type $\left[d_{j}, b\right]$, then the intersection clearly contains a or b. We may thus assume that $\mathrm{S}$ contains intervals of both types. We now define $\mathrm{d}$ by $\mathrm{d}$ $=\sup \left\{\mathrm{d}_{\mathrm{i}}\right\}$, and we complete the proof by showing that $\mathrm{d} \leq \mathrm{c}_{\mathrm{i}}$ for every i. Suppose that $\mathrm{c}_{\mathrm{i}_{0}}<\mathrm{d}$ for some $\mathrm{i}_{0}$. Then by the definition of $d$ there exists a $\mathrm{d}_{\mathrm{j}_{0}}$ such that $\mathrm{c}_{\mathrm{i}_{0}}<\mathrm{d}_{\mathrm{j}_{0}}$. Since $\left[\mathrm{a}, \mathrm{c}_{\mathrm{i}_{0}}\right] \cap\left[\mathrm{d}_{\mathrm{j}_{0}}, \mathrm{~b}\right]=\phi$, this contradicts the finite intersection property for $\mathrm{S}$ and concludes the proof.

Proposition 2.2 ${ }^{[9]}$ : (The Tube Lemma)

Let $\mathrm{X}_{1} \times \mathrm{X}_{2}$ be a product space where $\mathrm{X}_{2}$ is compact and let $\mathrm{p} \in \mathrm{X}_{1}$ be fixed. Let $\mathrm{N}$ be an nbd of the slice $\{\mathrm{p}\} \times \mathrm{X}_{2}$ in the product space. Then there exists an nbd $U$ of $P$ in $X_{1}$ such that $\{\mathrm{p}\} \times \mathrm{X}_{2} \subset \mathrm{U} \times \mathrm{X}_{2} \subset \mathrm{N}$ (The nbd $\mathrm{U} \times \mathrm{X}_{2}$ is called a tubular nbd or simply a tube).

Proposition 2.3 $3^{[9]}$ :

A finite product space $\mathrm{X}=\coprod_{\mathrm{j}=1}^{\mathrm{n}} \mathrm{X}_{\mathrm{j}}$ is compact iff each factor space $\mathrm{X}_{\mathrm{j}}$ is compact.

Note: The above result holds for arbitrary products also. The general result is known as the Tychonoff Theorem.

Theorem 2.11 ${ }^{[4]}$ : (The Generalized Heine-Borel Theorem)

Every closed and bounded subspace of $\mathbb{R}^{\mathrm{n}}$ is compact.

Theorem 2.12 $2^{[2]}$ : (Extreme value theorem)

Let $\mathrm{f}: \mathrm{X} \rightarrow \mathrm{Y}$ be continuous, where $\mathrm{Y}$ is an ordered set in the order topology. If $\mathrm{X}$ is compact, then there exit points $\mathrm{c}$ and $d$ in $X$ such that $f(c) \leq f(x) \leq f(d)$ for every $x \in X$.

The extreme value theorem of calculus is the special case of this theorem that occurs when we take $\mathrm{X}$ to be a closed interval in $\mathbb{R}$ and $\mathrm{Y}$ to be $\mathbb{R}$.

Definition:

A real number a $>0$ is called a Lebesgue number for our given open cover $\left\{\mathrm{G}_{\mathrm{i}}\right\}$ if each subset of $\mathrm{X}$ whose diameter is less than a is contained in at least one $G_{i}$.

\section{Definition:}

Let $\mathrm{X}$ be a topological space. If $\left(\mathrm{x}_{\mathrm{n}}\right)$ is a sequence of points of $X$, and if $n_{1}<n_{2}<\ldots \ldots \ldots \ldots \ldots<n_{i}<\ldots \ldots \ldots$.

is an increasing sequence of positive integers, then the sequence $\left(\mathrm{y}_{\mathrm{i}}\right)$ defined by setting $\mathrm{y}_{\mathrm{i}}=\mathrm{x}_{\mathrm{n}}$ is called a subsequence of the sequence $\left(x_{n}\right)$. The space $X$ is said to be sequentially compact if every sequence of points of $\mathrm{X}$ has a convergent subsequence.

Theorem 2.13 ${ }^{[4]}$ : (Lebesgue's Covering Lemma)

In a sequentially compact metric space, every open cover has a Lebesgue number.

Definition:

A space $X$ is said to be limit point compact if every infinite subset of $X$ has a limit point.

Note: On historical grounds, some call it "Frechet compactness"; others call it the "Bolzano weierstrass property."

Theorem $2.14^{[2]}$ :

Compactness implies limit point compactness, but not 
conversely.

Example:

Let $\mathrm{Y}$ consist of two points; give $\mathrm{Y}$ the topology consisting of $\mathrm{Y}$ and the empty set. Then the space $\mathrm{X}=\mathbb{Z}_{+} \times \mathrm{Y}$ is limit point compact, for every nonempty subset of $\mathrm{X}$ has a limit point. It is not compact, for the covering of $\mathrm{X}$ by the open sets $U_{n}=\{n\} \times Y$ has no finite subcollection covering $X$.

Now we prove that compactness and limit point compactness are equivalent in matrizable spaces.

Theorem 2.15 $5^{[2]}$ :

Let $\mathrm{X}$ be a metrizable space. Then the following are equivalent:

1. $\mathrm{X}$ is compact.

2. $\mathrm{X}$ is limit point compact.

3. $\mathrm{X}$ is sequentially compact.

Theorem 2.16 $6^{[4]}$ :

Any continuous mapping of a compact metric space into a metric space is uniformly continuous.

\section{Locally Compact Space}

Definition:

A space $X$ is said to be locally compact at $\mathrm{x}$ if there is some compact subspace $\mathrm{C}$ of $\mathrm{X}$ that contains a neighborhood of $\mathrm{X}$. if $\mathrm{X}$ is locally compact at each of its points, $\mathrm{X}$ is said simply to be locally compact.

Example :

Every compact space is locally compact, since a topological space is always a neighborhood of each of its points.

Example :

Every discrete space is locally compact, since for each point $\mathrm{p}$ in it, the singleton $\{\mathrm{p}\}$ is a compact nbd.

Example:

Every indiscrete space $\mathrm{X}$ is locally compact.

Example :

Consider the real line $\mathbb{R}$ with the usual topology. Observe that each point $p \in \mathbb{R}$ is interior to a closed interval, e.g. $[p-\delta$, $\mathrm{p}+\delta$ ] and that the closed interval is compact by the HeineBorel theorem. Hence $\mathbb{R}$ is locally compact space. On the other hand $\mathbb{R}$ is not a compact space. For example, the class

$\mathcal{A}=\{\ldots \ldots \ldots,(-3,-1),(-2,0),(-1,1),(0,2),(1,3), \ldots \ldots\}$ is an open cover of $\mathbb{R}$ but contains no finite subcover. Therefore $\mathbb{R}$ is locally compact but not compact.

Similarly $\mathbb{C}$ and $\mathbb{Z}$ are locally compact but not compact.

Theorem 3.1 ${ }^{[7]}$ :

Every closed subset of a locally compact space is locally compact.

Proof: Let B be a closed subset of a locally compact space $\mathrm{X}$ and let $\mathrm{x}$ be any point of $\mathrm{B}$. Then there exists a compact neighborhood $\mathrm{C}$ of $\mathrm{x}$. Therefore $\mathrm{B} \cap \mathrm{C}$ is a closed subset of C. Therefore $\mathrm{B} \cap \mathrm{C}$ is a compact subset of $\mathrm{C}$ (since every closed subset of a compact space is compact), and so $\mathrm{B} \cap \mathrm{C}$ is a compact subset of $B$. Thus $B \cap C$ is a compact neighborhood of $\mathrm{x}$ in the subspace $\mathrm{B}$ of $\mathrm{X}$. Hence $\mathrm{B}$ is locally compact.

Theorem $3.2^{[7]}$ :
Every separated locally compact space is regular Definition:

A subset $B$ of a topological space $X$ is said to be locally compact if $\mathrm{B}$ is locally compact considered as a subspace of $\mathrm{X}$.

\section{Theorem 3.3 $3^{[7]}$ :}

Let $\mathrm{x}$ be any point of a regular locally compact space X. Then the class consisting of all closed compact neighborhood of $x$ is a local base at $x$. The class consisting of all those open neighborhoods of $\mathrm{x}$ whose closures are compact is also a local base at $\mathrm{x}$.

Theorem 3.4 ${ }^{[7]}$ :

Let a subset $\mathrm{B}$ of a regular locally compact space $\mathrm{X}$ be the intersection of a closed and an open subset of X. Then B is locally compact.

Proof: Let $\mathrm{x}$ be any point of $\mathrm{B}$. Then there is a neighborhood $\mathrm{N}$ of $\mathrm{x}$ such that $\mathrm{B} \cap \mathrm{N}$ is a closed subset of the subspace $\mathrm{N}$ of $\mathrm{X}$. Since $\mathrm{X}$ is regular and locally compact, then by the previous theorem there is a compact neighborhood $K$ of $x$ such that $K \subset N$. Since $B \cap N$ is a closed subset of the subspace $\mathrm{N},(\mathrm{B} \cap \mathrm{N}) \cap \mathrm{K}$ is a closed subset of K. Since K is compact,

$(\mathrm{B} \cap \mathrm{N}) \cap \mathrm{K}$ is compact. Therefore $(\mathrm{B} \cap \mathrm{N}) \cap \mathrm{K}=\mathrm{B} \cap \mathrm{K}$ is a compact neighborhood of $\mathrm{x}$ in the subspace $\mathrm{B}$ of $\mathrm{X}$. Hence B is locally compact.

Theorem $3.5^{[3]}$ :

If $U$ is a neighborhood of a closed compact subset $A$ of a regular locally compact topological space $X$, then there is a closed compact neighborhood $\mathrm{V}$ of $\mathrm{A}$ such that $\mathrm{A} \subset \mathrm{V} \subset \mathrm{U}$.

Moreover, there is a continuous function $\mathrm{f}$ on $\mathrm{X}$ to the closed unit interval such that $\mathrm{f}$ is zero on $\mathrm{A}$ and one on $\mathrm{X}-\mathrm{V}$.

Proof:

For each point $\mathrm{x}$ of $\mathrm{A}$ there is a neighbourhood $\mathrm{W}$ which is closed compact subset of U. Since A is compact it may be covered by a finite family of such neighbourhoods and their union is a closed compact neighbourhood V of $\mathrm{A}$. Then $\mathrm{V}$ with the relative topology is a regular compact space which is therefore normal. Hence there is a continuous function $g$ on $\mathrm{V}$ to the closed unit interval such that $\mathrm{g}$ is zero on $\mathrm{A}$ and one on $\mathrm{V}-\mathrm{V}^{0}\left(\mathrm{~V}^{0}\right.$ is the interior of $\left.\mathrm{V}\right)$. Let $\mathrm{f}$ equal $\mathrm{g}$ on $\mathrm{V}$ and one on

$\mathrm{X}-\mathrm{V}$. Then $\mathrm{f}$ is continuous because $\mathrm{V}^{0}$ and $\mathrm{X}-\mathrm{V}$ are separated and $\mathrm{f}$ is continuous on $\mathrm{V}$ and $\mathrm{X}-\mathrm{V}^{0}$.

Theorem 3.6 $6^{[7]}$ :

A regular locally compact space is completely regular.

Theorem 3. $7^{[7]}$ :

A subset $\mathrm{B}$ of a locally compact space $\mathrm{X}$ is closed if $\mathrm{B} \cap \mathrm{C}$ is closed for every compact subset $\mathrm{C}$ of $\mathrm{X}$.

Proof:

Let $\mathrm{B} \cap \mathrm{C}$ be closed for every compact subset $\mathrm{C}$ of $\mathrm{X}$. Let $\mathrm{X}$ be any contact point of $\mathrm{B}$. Since $\mathrm{X}$ is locally compact, there exists a compact neighbourhood $\mathrm{C}$ of $\mathrm{x}$. Now let $\mathrm{N}$ be any neighbourhood of $\mathrm{x}$.

We know that, if $x$ is any point of topological space $X$, then the class $\mathrm{v}(\mathrm{x})$ of neighbourhoods of $\mathrm{x}$. If $\mathrm{M}$ and $\mathrm{N}$ are members of $\mathrm{v}(\mathrm{x})$ then $\mathrm{M} \cap \mathrm{N}$ is also a member of $\mathrm{v}(\mathrm{x})$. Hence by the above theorem $\mathrm{C} \cap \mathrm{N}$ is a neighbourhood of $\mathrm{x}$, 
and as such $(\mathrm{C} \cap \mathrm{N}) \cap \mathrm{B}$ is non void. Hence $\mathrm{x}$ is a contact point of $\mathrm{B} \cap \mathrm{C}$. But $\mathrm{B} \cap \mathrm{C}$ is closed.

Also we know that a subset $B$ of a topological space is closed if and only if $\mathrm{B}=\overline{\mathrm{B}}$. Hence from the above $\mathrm{x}$ is in $\mathrm{B}$ $\cap \mathrm{C}$ and therefore in B. Thus B is closed.

Theorem $3.8^{[3]}$ :

If $\mathrm{X}$ is a locally compact topological space which is either Hausdorff or regular, then the family of closed compact neighbourhoods of each point is a base its neighbourhood system.

Proof:

Let $\mathrm{x}$ be a point of $\mathrm{X}, \mathrm{C}$ a compact neihgbourhood of $\mathrm{x}$, and $\mathrm{U}$ an arbitrary neihgbourhood of $\mathrm{x}$. If $\mathrm{X}$ is regular, then there is a closed neihgbourhood $\mathrm{V}$ of $\mathrm{x}$ which is a subset of the intersection of $U$ and the interior of $C$, and evidently $\mathrm{V}$ is closed and compact. If $\mathrm{X}$ is Hausdorff and $\mathrm{W}$ is the interior of UnC, then, since $\overline{\mathrm{W}}$ is a compact Hausdorff space, W contains a closed compact set $\mathrm{V}$ which is a neighborhood of $\mathrm{x}$ in $\overline{\mathrm{W}}$; but $\mathrm{V}$ is also a neighborhood of $\mathrm{x}$ in $\mathrm{W}$ (that is, with respect to the relativized topology for $\mathrm{W}$ ) and is therefore a neighborhood of $\mathrm{x}$ in $\mathrm{X}$.

Note: In particular it follows that every locally compact Hausdorff space is regular; actually a stronger statement is true.

Proposition 3.1 $1^{[8]}$ :

An open subspace of a locally compact, regular space is locally compact.

Proof: Let $\mathrm{X}$ be locally compact and regular and suppose $\mathrm{Y}$ is open in $\mathrm{X}$. Let $\mathrm{y} \in \mathrm{Y}$. Then $\mathrm{Y}$ is a neighborhood of $\mathrm{y}$ in $\mathrm{X}$ and so there exists a compact neighborhood $\mathrm{C}$ of $\mathrm{y}$ such that $\mathrm{C} \subset \mathrm{Y}$. But then, $\mathrm{Y}$ is locally compact at $\mathrm{y}$. Since $\mathrm{y}$ was an arbitrary point of $\mathrm{Y}$, it follows that $\mathrm{Y}$ is locally compact in the relative topology on it.

Proposition 3.2 $2^{[8]}$ :

Let $\mathrm{X}$ be a Hausdorff space and $\mathrm{Y}$ be a dense subset of $\mathrm{X}$. If $\mathrm{Y}$ is locally compact in the relative topology on it, then $\mathrm{Y}$ is open in $\mathrm{X}$.

Theorem 3. $9^{[8]}$ :

A subspace of a locally compact, Hausdorff space is locllay compact iff it is open in its closure.

Proof: Let $\mathrm{X}$ be a locally compact Hausdorff space and $\mathrm{Y}$ be a subspace of $X$. Put $Z=\bar{Y}$. Then $Z$ itself is locally compact for it is easy to show that local compactness is weakly hereditary. Also Z is Hausdorff and therefore regular (Since every locally compact, Hausdorff space is regular).

Now if $\mathrm{Y}$ is open in $\mathrm{Z}$ then $\mathrm{Y}$ is locally compact by proposition 1 .

Conversely if $\mathrm{Y}$ is locally compact, then by the last proposition applied to $\mathrm{Z}$, we see that $\mathrm{Y}$ is open in $\mathrm{Z}$.

Theorem 3.10 ${ }^{[10]}$ :

The product of finitely many locally compact spaces is a locally compact space.

Proof: Let $\mathrm{S}_{1}, \mathrm{~S}_{2}, \ldots \ldots \ldots$, and $\mathrm{S}_{\mathrm{n}}$ each be locally compact, and let $\mathrm{p}=\left(\mathrm{p}_{1}, \ldots \ldots \ldots, \mathrm{p}_{\mathrm{n}}\right)$ be any point in $\mathrm{S}_{1} \times \mathrm{S}_{2} \times \ldots \ldots \times \mathrm{S}_{\mathrm{n}}$. In each space $S_{i}$ there is an open set $U_{i}$ containing $p_{i}$ and having compact closure. Then the basis element $\mathrm{U}_{1} \times \mathrm{U}_{2} \times \ldots . \times \mathrm{U}_{\mathrm{n}}$ contains $\mathrm{p}$ and has closure
$\overline{\mathrm{U}_{1}} \times \overline{\mathrm{U}_{2}} \times \ldots \ldots . . \overline{\mathrm{U}_{\mathrm{n}}}$ which is compact by the Tychonoff theorem. Hence

$\mathrm{S}_{1} \times \mathrm{S}_{2} \times \ldots \ldots \times \mathrm{S}_{\mathrm{n}}$ is locally compact.

Theorem 3.11 $1^{[3]}$ :

If a product is locally compact then each coordinate space is locally compact and all except a finite number of coordinate spaces are compact.

Proof:

If a product is locally compact, then each coordinate space is locally compact because projection into a coordinate space is open.

We know that if infinitely many coordinate spaces are noncompact, then each compact subset of the product is nowhere dense, and no point has a compact neighbourhood.

\section{Compactification}

Definition:

Let $\mathrm{X}$ be a locally compact Hausdorff space. Take some object outside $\mathrm{X}$, denoted by the symbol $\infty$ for convenience, and adjoin it to $X$, forming the set $Y=X \cup\{\infty\}$.

Topologize $\mathrm{Y}$ by defining, the collection of open sets in $\mathrm{Y}$ to be all sets of the following types:

(1) U, where U is an open subset of $X$,

(2) $Y-C$, where $C$ is compact subset of $X$.

The space $\mathrm{Y}$ is called the one-point compactification of $\mathrm{X}$. We need to check that this collection of open sets is a topology on Y.

The empty set is a set of type of (1), and the space $\mathrm{Y}$ is set of type (2). Checking that the intersection of two open sets is open involves three cases:

$\mathrm{U}_{1} \cap \mathrm{U}_{2}$ is of the type (l).

$\left(\mathrm{Y}-\mathrm{C}_{1}\right) \cap\left(\mathrm{Y}-\mathrm{C}_{2}\right)=\mathrm{Y}-\left(\mathrm{C}_{1} \cup \mathrm{C}_{2}\right)$ is of the type (2).

$\mathrm{U}_{1} \cap\left(\mathrm{Y}-\mathrm{C}_{1}\right)=\mathrm{U}_{1} \cap\left(\mathrm{X}-\mathrm{C}_{1}\right)$ is of the type (1), because $\mathrm{C}_{1}$ is closed in $\mathrm{X}$.

Similarly, one checks that the union of any collection of open sets is open:

$\mathrm{U} \mathrm{U}_{\alpha}=\mathrm{U}$ is of the type (1)

$\mathrm{U}\left(\mathrm{Y}^{\alpha}-\mathrm{C}_{\beta}\right)=\mathrm{Y}-\left(\cap \mathrm{C}_{\beta}\right)=\mathrm{Y}-\mathrm{C}$ is of type (2)
$\left(\mathrm{U} \mathrm{U}_{\alpha}\right) \cup\left(\mathrm{U}\left(\mathrm{Y}-\mathrm{C}_{\beta}\right)\right)=\mathrm{U} \cup(\mathrm{Y}-\mathrm{C})=\mathrm{Y}-(\mathrm{C}-\mathrm{U})$

which is of the type (2),

because $(\mathrm{C}-\mathrm{U})$ is a closed subset of $\mathrm{C}$ and therefore compact.

\section{Example :}

The one point compactification of the real line $\mathbb{R}$ is homeomorphic with the circle. Similarly, the one-point compactification of $\mathbb{R}^{2}$ is homemorphic to the sphere $\mathrm{S}^{2}$.

Theorem 4.1 $1^{[2]}$ :

Let $\mathrm{X}$ be a locally compact Hausdorff space, which is not compact; Let $\mathrm{Y}$ be the one point Compactification of $\mathrm{X}$. Then $\mathrm{Y}$ is a compact Hausdorff space; $\mathrm{X}$ is a subspace of $\mathrm{Y}$; the set $\mathrm{Y}-\mathrm{X}$ consists of a single point; and

$\operatorname{cl}(\mathrm{X})=\mathrm{Y}$.

Theorem 4.2 ${ }^{[3]}$ : (ALEXANDROFF)

The one-point compactification $\mathrm{X}^{*}$ of a topological space $\mathrm{X}$ is compact and $\mathrm{X}$ is a subspace. The space $\mathrm{X}^{*}$ is Hausdorff 
if and only if $\mathrm{X}$ is locally compact and Hausdorff.

Proof: A set $U$ is open in $X^{*}$ iff (a) $U \cap X$ is open in $X$ and (b) whenever $\infty \in U$, then $X-U$ is compact. Consequently finite intersections and arbitrary unions of sets open in $X^{*}$ intersect $X$ in open sets. If $\infty$ is a member of the intersection of two open subsets of $X^{*}$, then the complement of the intersection is the union of two closed compact subsets of $X$ and is therefore closed and compact. If $\infty$ belongs to the union of the members of a family of open subsets of $X^{*}$, then $\infty$ belongs to some member $U$ of the family, and the complement of the union is a closed subset of the compact set $\mathrm{X}-U$ and is therefore closed and compact. Consequently $X^{*}$ is a topological space and $\mathrm{X}$ is a subspace. If $U$ is an open cover of $X^{*}$, then $\infty$ is a member of some $U$ in $U$ and $\mathrm{X}-U$ is compact, and hence there is a finite subcover of $U$. Therefore $\mathrm{X}^{*}$ is compact. If $X^{*}$ is a Hausdorff space, then its open subspace $X$ is a locally compact Hausdorff space. Finally it must be shown that $X^{*}$ is a Hausdorff space if $\mathrm{X}$ is a locally compact Hausdorff space. It is only necessary to show that, if. $x \in \mathrm{X}$, then there are disjoint neighborhoods of $x$ and $\infty$. But since $\mathrm{X}$ is locally compact and Hausdorff there is a closed compact neighbourhood $U$ of $x$ in $X$ and $X^{*}-U$ is the required neighbourhood of $\infty$.

Theorem 4.3 $3^{[7]}$ : A one-point compaciificalion of a topological space $A$ is separated iff $\mathrm{A}$ is separated and locally compact.

Lemma 4.1 $1^{[2]}$ :

Let $\mathrm{X}$ be Hausdorff space. Then $\mathrm{X}$ is locally compact at $\mathrm{X}$ if and only if for every neighbourhood $U$ of $x$, there is a neighbourhood $\mathrm{V}$ of $\mathrm{x}$ such that $\mathrm{cl}(\mathrm{V})$ is compact and $\mathrm{cl}(\mathrm{V})$ $\subset \mathrm{U}$.

Corollary $4.1^{[2]}$ :

Let $\mathrm{X}$ be a locally compact Hausdorff space and $\mathrm{Y}$ be a subspace of $\mathrm{X}$. If $\mathrm{Y}$ is closed in $\mathrm{X}$ or opens in $\mathrm{X}$, then $\mathrm{Y}$ is locally compact.

Proof: Suppose that $\mathrm{Y}$ is closed in $\mathrm{X}$. Given $\mathrm{y} \in \mathrm{Y}$, let $\mathrm{C}$ be a compact set in $\mathrm{X}$ containing the neighbourhood $\mathrm{U}$ of $\mathrm{X}$ in $\mathrm{X}$. Then $\mathrm{C} \cap \mathrm{Y}$ is closed in $\mathrm{C}$ and thus compact. Then it contains the neighbourhood $\mathrm{U} \cap \mathrm{Y}$ of $\mathrm{y}$ in $\mathrm{Y}$. (We have not used the Hausdorff condition here.)

Again suppose that $\mathrm{Y}$ is open in $\mathrm{X}$. Given $\mathrm{y} \in \mathrm{Y}$, we apply the previous lemma to choose a neighbourhood $\mathrm{V}$ of $\mathrm{y}$ in $\mathrm{X}$ such that $\mathrm{cl}(\mathrm{V})$ is compact and $\operatorname{cl}(\mathrm{V}) \subset \mathrm{U}$. Then $\mathrm{C}=\mathrm{cl}(\mathrm{V})$ is a compact set in $\mathrm{Y}$ containing the neighbourhood $\mathrm{V}$ of $\mathrm{y}$ in $\mathrm{Y}$. Hence the proof of the theorem is complete.

Corollary $4.2^{[2]}$ :

A space $\mathrm{X}$ is homeomorphic to an open subset of a compact Hausdorff space if and only if $\mathrm{X}$ is locally compact Hausdorff.

Definition: A compactification of a space $\mathrm{X}$ is a compact Hausdorff space $\mathrm{Y}$ containing $\mathrm{X}$ such that $\mathrm{X}$ is dense in $\mathrm{Y}($ That is, such that $\mathrm{cl}(\mathrm{X})=\mathrm{Y})$.

Two compactification $Y_{1}$ and $Y_{2}$ are said to be equivalent if there is a homeomorphism $\mathrm{h}: \mathrm{Y}_{1} \rightarrow \mathrm{Y}_{2}$ such that $\mathrm{h}(\mathrm{x})=\mathrm{x}$ for every $\mathrm{x} \in \mathrm{X}$.

In order for $\mathrm{X}$ to have a compactification, $\mathrm{X}$ must be completely regular, because a subset of a compact Hausdorff space is necessarily completely regular.

Conversely, every completely regular space has at least one compactification.

One way of obtaining a compactification of $\mathrm{X}$ is as follows:

Suppose $\mathrm{X}$ is completely regular space. Choose an imbedding

h: $X \rightarrow Z$ of $X$ in a compact Hausdorff space $Z$. Let $X_{0}$ denote the subspace $h(X)$ of $Z$, and let $Y_{0}$ denote its closure in $Z$. Then $Y_{0}$ is a compact Hausdorff space and

$\mathrm{c} 1\left(\mathrm{X}_{0}\right)=\mathrm{Y}_{0}$; Therefore, $\mathrm{Y}_{0}$ is compactification of $\mathrm{X}_{0}$.

Example:

Let $\mathrm{Y}$ be the space $[0,1]$. Then $\mathrm{Y}$ is compactification of $\mathrm{X}$ $=(0,1)$. It is obtained by "adjoining one point at each end of $(0,1) . ”$

\section{Definition:}

Let $\mathrm{X}$ be a completely regular space. Let $\left\{\mathrm{f}_{\alpha}\right\}_{\alpha \in \mathrm{J}}$ be the collection of all bounded continuous real valued functions on $\mathrm{X}$, indexed by some index set $\mathrm{J}$. For each $\alpha \in \mathrm{J}$, choose a closed interval $I_{\alpha}$ in $\mathbb{R}$ containing $\mathrm{f}_{\alpha}(\mathrm{X})$. To be definite, choose $\mathrm{I}_{\alpha}=\left[\mathrm{glb} \mathrm{f}_{\alpha}(\mathrm{X}), \operatorname{lub~f}_{\alpha}(\mathrm{X})\right]$.

Then define, $\mathrm{h}: \mathrm{X} \rightarrow \prod_{\mathrm{I}_{\alpha}}$ by the rule $\mathrm{h}(\mathrm{x})=\left(\mathrm{f}_{\alpha}(\mathrm{x})\right)_{\alpha \in \mathrm{J}}$. Then by Tychonoff theorem $\prod_{I_{\alpha}}$ is compact. Since $X$ is completely regular, the collection $\left\{\mathrm{f}_{\alpha}\right\}$ separates points from closed sets in $\mathrm{X}$. Therefore, by the Imbedding theorem $\mathrm{h}$ is an imbedding. The compactification of $X$ induced by $h$ is called the Stone-Cech compactification of X. it is denoted by $\beta(X)$.

The property of the Stone-Cech compactification is the following:

Theorem $4.4^{[2]}$ :(Extension property)

Let $\mathrm{X}$ be a completely regular space and let $\beta(\mathrm{X})$ be its Stone-Cech compactification. Then every bounded continuous real-valued function on $\mathrm{X}$ can be uniquely extended to a continuous real-valued function on $\beta(X)$.

Uniqueness of the extension is a consequence of the following lemma:

\section{Lemma $4.2^{[2]}$ :(Uniqueness of the extension)}

Let $\mathrm{A} \subset \mathrm{X}$; let $\mathrm{f}: \mathrm{A} \rightarrow \mathrm{Z}$ be a continuous map of $\mathrm{A}$ in to the Hausdorff space $Z$. There is at most one extension of $f$ to a continuous function $\mathrm{g}: \mathrm{cl}(\mathrm{A}) \rightarrow \mathrm{Z}$

Proof: Suppose that $\mathrm{g}, \mathrm{g}^{\prime}: \mathrm{cl}$ (A) $\rightarrow \mathrm{X}$ are two different extensions of $\mathrm{f}$; Choose $\mathrm{x}$ so that $\mathrm{g}(\mathrm{x}) \neq \mathrm{g}^{\prime}(\mathrm{x})$. Let $\mathrm{U}$ and $\mathrm{U}^{\prime}$ be disjoint neighbourhoods of $\mathrm{g}(\mathrm{x})$ and $\mathrm{g}^{\prime}(\mathrm{x})$, respectively. Again choose a neighbourhood $\mathrm{V}$ of $\mathrm{x}$ so that $\mathrm{g}(\mathrm{V}) \subset \mathrm{U}$ and $\mathrm{g}^{\prime}(\mathrm{V}) \subset \mathrm{U}^{\prime}$. Now $\mathrm{V}$ intersects $\mathrm{A}$ in some point $\mathrm{y}$; then $\mathrm{g}(\mathrm{y}) \in \mathrm{U}$ and $\mathrm{g}^{\prime}(\mathrm{y}) \subset \mathrm{U}^{\prime}$. But since $\mathrm{y} \in \mathrm{A}$, we have $\mathrm{g}(\mathrm{y})=$ $\mathrm{f}(\mathrm{y})$ and $g^{\prime}(\mathrm{y})=\mathrm{f}(\mathrm{y})$. This contradicts the fact that $U$ and $\mathrm{U}^{\prime}$ are disjoint. This completes the proof of the theorem.

The following theorem characterized the fact that StoneCech compactification in a space is unique.

Theorem $4.5^{[2]}$ :

Let $\mathrm{X}$ be completely regular. Let $\mathrm{Y}_{1}$ and $\mathrm{Y}_{2}$ be two compactification of $X$ having the extension property. Thus there is a homeomorphism $\Phi$ of $Y_{1}$ onto $Y_{2}$ such that $\Phi(\mathrm{x})$ 
$=\mathrm{x}$ for each $\mathrm{x} \in \mathrm{X}$

\section{5. $\sigma$-Compact and Finally Compact Spaces}

Definition:

A topological space $\mathrm{X}$ is said to be $\sigma$-compact if and only if $\mathrm{X}$ is the union of a countable family of its compact subsets. Theorem 5.1 ${ }^{[7]}$ :

Every closed subset of a $\sigma$-compact space is $\sigma$-compact.

Proof: Let $\mathrm{C}$ be any closed subset of a $\sigma$-compact space $\mathrm{X}$. Then $X$ is the union of a countable family $\left\{\mathrm{K}_{\mathrm{n}}: \mathrm{n} \in \mathbb{N}\right\}$ of its compact subsets. Hence

$C \cap K_{n}$ is a closed subset of the subspace $K_{n}$ of $X$ for each $\mathrm{n} \in \mathbb{N}$. Hence

$\mathrm{C} \cap \mathrm{K}_{\mathrm{n}}$ is compact in $\mathrm{C}$ for each $\mathrm{n} \in \mathbb{N}$. Consequently $\mathrm{C}$ is the union of the countable family $\left\{C \cap \mathrm{K}_{\mathrm{n}}: \mathrm{n} \in \mathbb{N}\right\}$ of its compact subsets. Thus $\mathrm{C}$ is

$\sigma$-compact.

Theorem 5.2 $2^{[7]}$.

Every separated locally compact space $\mathrm{X}$ with a countable open base is $\sigma$-compact.

Proof: Let $\alpha$ be any countable open base for X. Let $\mathrm{x}$ be any point of $\mathrm{X}$. Then $\mathrm{x}$ has a compact closed neighbourhood $\mathrm{K}$. Consequently there exists

a member $\mathrm{M}$ of $\alpha$ such that $\mathrm{x} \in \mathrm{M} \subset \mathrm{K}$.

We know that if $\mathrm{B}$ and $\mathrm{C}$ are any subsets of a topological space $\mathrm{X}$ and if $\mathrm{B} \subset \mathrm{C}$ then $\overline{\mathrm{B}} \subseteq \overline{\mathrm{C}}$ and we also know that a subset $\mathrm{B}$ of a topological space $\mathrm{X}$ is closed if and only if $\mathrm{B}=$ $\bar{B}$. Since $K$ is closed, hence from the above theorem we have $\bar{M} \subset K$. Since $K$ is a separated compact subspace of $X$ and we know that every compact subset of a separated space is closed. Hence from the above $\bar{M}$ is a compact subset of $\mathrm{K}$ and so of $\mathrm{X}$. Thus for each $\mathrm{x}$ in $\mathrm{X}$ there is a member $\mathrm{M}$ of the countable open base $\alpha$ such that $x \in \bar{M}$. It follows that $X$ is the union of the countable family $\{\bar{M}: M \in \alpha\}$ of compact subsets.

Theorem 5.3 $3^{[7]}$ :

If $\mathrm{X}$ is a $\sigma$-compact locally compact separated space then there exists a sequence $\left(B_{n}: n \in \mathbb{N}\right)$ of compact subsets of $X$ with the property that $\left(B_{n}^{0}: n \in \mathbb{N}\right)$ covers $X$ and for each compact subset $\mathrm{C}$ of $\mathrm{X}$ there is an $\mathrm{n}$ in $\mathbb{N}$ such that $\mathrm{C} \subset \mathrm{B}_{\mathrm{n}}^{0}$.

Theorem 5.4 ${ }^{[7]}$ :

Let $\mathrm{X}$ be a locally compact separated space and let $\mathrm{X}^{*}=\mathrm{X}$ $\cup\{\infty\}$ be a one-point compactification of X. Then $X$ is $\sigma$ compact if $\infty$ has a countable local base in $\mathrm{X}^{*}$.

Definition:

A topological space $\mathrm{X}$ is said to be finally compact or a Lindeloff space if and only if every open cover of $\mathrm{X}$ has a countable subcover.

A subset $\mathrm{B}$ of $\mathrm{X}$ is said to be finally compact if and only if $\mathrm{B}$ is finally compact as a subspace of $\mathrm{X}$.

Theorem 5. $5^{[7]}$ :

Every topological space with a countable open base is finally compact.
Proof: Let $\beta$ be an open cover of a topological space $\mathrm{X}$ with a countable open base $\gamma$. Then for each $\mathrm{x}$ in $\mathrm{X}$ there is a member $B$ of $\beta$ such that $x \in B$ and we know that a subclass $\beta$ of a topology $\mathcal{T}$ for a set $X$ is a base of $\mathcal{T}$ if and only if for each $\mathrm{U}$ in $\mathcal{T}$ and each $\mathrm{x}$ in $\mathrm{U}$ there is a member $\mathrm{V}$ of $\beta$ such that $\mathrm{x} \in \mathrm{V} \subset \mathrm{U}$. Hence from the above theorem, there is a member $C_{x}$ of $V$ such that $x \in C_{x} \subset B$. Consequently the subclass $\left\{C_{x}: x \in X\right\}$ of $\gamma$ is a countable cover of $X$. For each $C_{x}$ we can pick a member $B_{x}$ of $\beta$ such that $C_{x} \subset B_{x}$. Then $\left\{B_{x}: x \in X\right\}$ is a countable subcover of $\beta$. Hence $X$ is finally compact.

Theorem 5. $6^{[7]}$ :

Every $\sigma$-compact space is finally compact.

Proof: Let $\mathrm{X}$ be a $\sigma$-compact space. Then there is a countable family

$\left\{X_{n}: n \in \mathbb{N}\right\}$ of compact subsets of $X$ such that $X=\bigcup\left\{X_{n}\right.$ : $\mathrm{n} \in \mathbb{N}\}$. Consider any open cover $\alpha$ of $X$. For each $n$ in $\mathbb{N}$ there is a finite subclass $\alpha_{n}$ of $\alpha$ covering $X_{n}$, because $X_{n}$ is compact. Hence $U\left\{\alpha_{n}: n \in \mathbb{N}\right\}$ is a countable subclass of $\alpha$ and covers $\mathrm{X}$. Hence $\mathrm{X}$ is finally compact.

Theorem 5. $7^{[7]}$ :

Every regular finally compact space is normal

Proof:

Let $\mathrm{A}$ and $\mathrm{B}$ be any two disjoint closed subsets of a regular finally compact space $X$. Since $X$ is regular, there exists an open set $\mathrm{U}_{\mathrm{x}}$ for each $\mathrm{x}$ in $\mathrm{A}$ such that $\mathrm{x} \in \mathrm{U}_{\mathrm{x}} \subset \overline{\mathrm{U}}_{\mathrm{x}} \subset(\mathrm{X}-\mathrm{B})$. The class $\left\{U_{x}: x \in A\right\}$ covers $A$. Therefore $\left\{U_{x}: x \in A\right\} \cup$ $\{X-A\}$ is an open cover of $X$. Since $X$ is finally compact, there exits a countable subclass of this cover covering $\mathrm{X}$ and therefore $\mathrm{A}$, and the closure of each member of this countable class is disjoint from B. By an analogous argument there exists a countable class of open subsets of $X$ such that this class covers $\mathrm{B}$ and the closures of each member of which is disjoint form A. Hence A and $B$ have disjoint neighbourhoods. Thus $\mathrm{X}$ is normal.

\section{Conclusion}

In this study we show that compactness, limit point compactness and sequentially compactness are equivalent in metrizable spaces. We introduce it here as an interesting application of the Tychonoff theorem. We show that every compact space is locally compact but not conversely. We also show that the product of finitely many compact spaces is a locally compact space. We show that Stone-Cech compactification in a space is unique. We also show that every regular finally compact space is normal. This research work would give some remarkable results which can be used to study the whole topological space by studying a finite number of open sets.

\section{References}

[1] Seymour Lipschutz, General Topology, McGraw-Hill Book Company, Singapore, 1965.

[2] Munkres, James R, Topology, 2nd edition, Prentice Hall, 2000. 
[3] John L. Kelley, General topology, Van Nostrand, 1955.

[4] George F. Simmons, Topology and Modern Analysis, McGraw-Hill, Inc. 1963.

[5] Mitra, M., Study of some properties of topological spaces and of their generalizations, Ph.D. Thesis, Rajshahi University, 2006.

[6] Bert Mendelson, Introduction to Topology, Allynand and Bacon, Inc. U.S.A., 1985.

[7] N.D. Gautam and Shanti Narayan, General Topology, 1976.
[8] K.D. Joshi, Introduction to General Topology, 1983

[9] J V Deshpabnde, Introductory to Topology,Centre of advanced study in Mathematics, University of Bombay.

[10] John G Hocking and Gall S Young, Topology, Addison Weslesy, New York, 1961

[11] Steven A. Gall, Point Set Topology, 1964.

[12] Dugundji, J., Topology, Wm. C. Brown Publisher, 1989 (Reprint New Delhi, 1995). 